\title{
Intravitreal dexamethasone in exogenous bacterial endophthalmitis: results of a prospective randomised study
}

Taraprasad Das, Subhadra Jalali, Vijaya K Gothwal, Savitri Sharma, Thomas J Naduvilath

\begin{abstract}
Aim-To evaluate the efficacy of intravitreal dexamethasone co-administered with intravitreal antibiotics along with vitrectomy in the management of exogenous bacterial endophthalmitis.

Methods-In a prospective randomised clinical trial, 63 patients (63 eyes) with suspected bacterial endophthalmitis (postoperative and post-traumatic) were treated with vitrectomy and intravitreal antibiotics and randomised to intravitreal dexamethasone $($ IOAB with $=29$ eyes) and no dexamethasone (IOAB without $=34$ eyes). Inflammation score (IS) and visual acuity were measured by two masked observers before surgery, and at 1,4 , and 12 weeks after surgery in both the groups.

Results-There was significant reduction $(p<0.0001)$ in IS at 1,4 , and 12 weeks after the surgery in the "IOAB with" group; there was temporary but significant increase $(p<0.01)$ in IS at 1 week in the "IOAB without" group, before decline (p $<0.001$ ) of IS at 4 and 12 weeks. The magnitude and relative percentage change in IS between the two groups were found to be significant at $1(\mathrm{p}<0.0001)$, and 4 (p $<0.01$ ) weeks, and not at 12 weeks. The visual acuity at 12 weeks was comparable in both the IOAB with and IOAB without groups.
\end{abstract}

Conclusion-Intravitreal dexamethasone helps in early reduction of inflammation in exogenous bacterial endophthalmitis, but has no independent influence on the visual outcome. In selected patients with endophthalmitis where oral corticosteroids cannot be given for medical reasons intravitreal corticosteroids could be beneficial; in other situations they could be complementary to oral corticosteroid therapy.

(Br f Ophthalmol 1999;83:1050-1055)

Corticosteroids are recommended as an important adjunct to antibiotics and vitrectomy in the management of infectious bacterial endophthalmitis. ${ }^{1-6}$ The corticosteroids are known to limit the degree of inflammation caused by toxins liberated from the microorganisms. The Endophthalmitis Vitrectomy Study (EVS) recommends oral prednisolone (1 $\mathrm{mg} / \mathrm{kg}$ body weight), starting a day after the intraocular antibiotic (IOAB) therapy, with or without vitrectomy in patients with post cataract or secondary IOL endophthalmitis. ${ }^{78}$
Oral corticosteroid, however, is not without side effects; on occasions it could be difficult to initiate or continue the therapy, or may require discontinuation of treatment. Intravitreal corticosteroids have also been shown to limit the degree of inflammation of the vitreous and the surrounding ocular tissues without any toxicity to the retina in bacterial endophthalmitis. ${ }^{1-6}$ Considering the side effects of systemic corticosteroids, it is worth exploring clinically the role of intravitreal corticosteroids in bacterial endophthalmitis, so that one could exclusively use this treatment in selected situations where systemic steroids can not be administered for medical reasons. Keeping such situations in mind, a prospective randomised clinical study was designed to evaluate both objectively and subjectively, the safety and efficacy of intravitreal corticosteroids in non-fungal postoperative and post-traumatic endophthalmitis.

The issues regarding the use of intravitreal corticosteroids are:

(1) Do they limit the ocular inflammation?

(2) Do they interfere with the infection control actions of the IOAB?

(3) Is the final visual recovery affected?

The outcome measures of the study were the extent and speed of reduction of inflammation, detectable toxicity of the retina (based on the symptoms, ophthalmoscopy, fluorescein angiography), and the visual acuity at both 1 and 3 months postoperatively.

\section{Materials and methods}

All patients referred to the retina vitreous service of the institute over 24 months (January 1993 to December 1994) with suspected bacterial endophthalmitis were enrolled into the study. Informed consent from the patient (or the family in the case of minors) was obtained for vitrectomy, and IOAB with or without intravitreal dexamethasone. The nature of the study was explained and discussed with the patient and the family available at the time of recruitment; it was also explained that should it be considered an absolute necessity to administer oral corticosteroid at any time postoperatively, the patient will be withdrawn from the study, and oral steroids will then be given. The study was approved by the institutional review board.

STUDY DESIGN

The study design in shown in Figure 1. The baseline evaluation in all the patients consisted of a detailed history including trauma or previ-
Accepted for publication 31 May 1999 


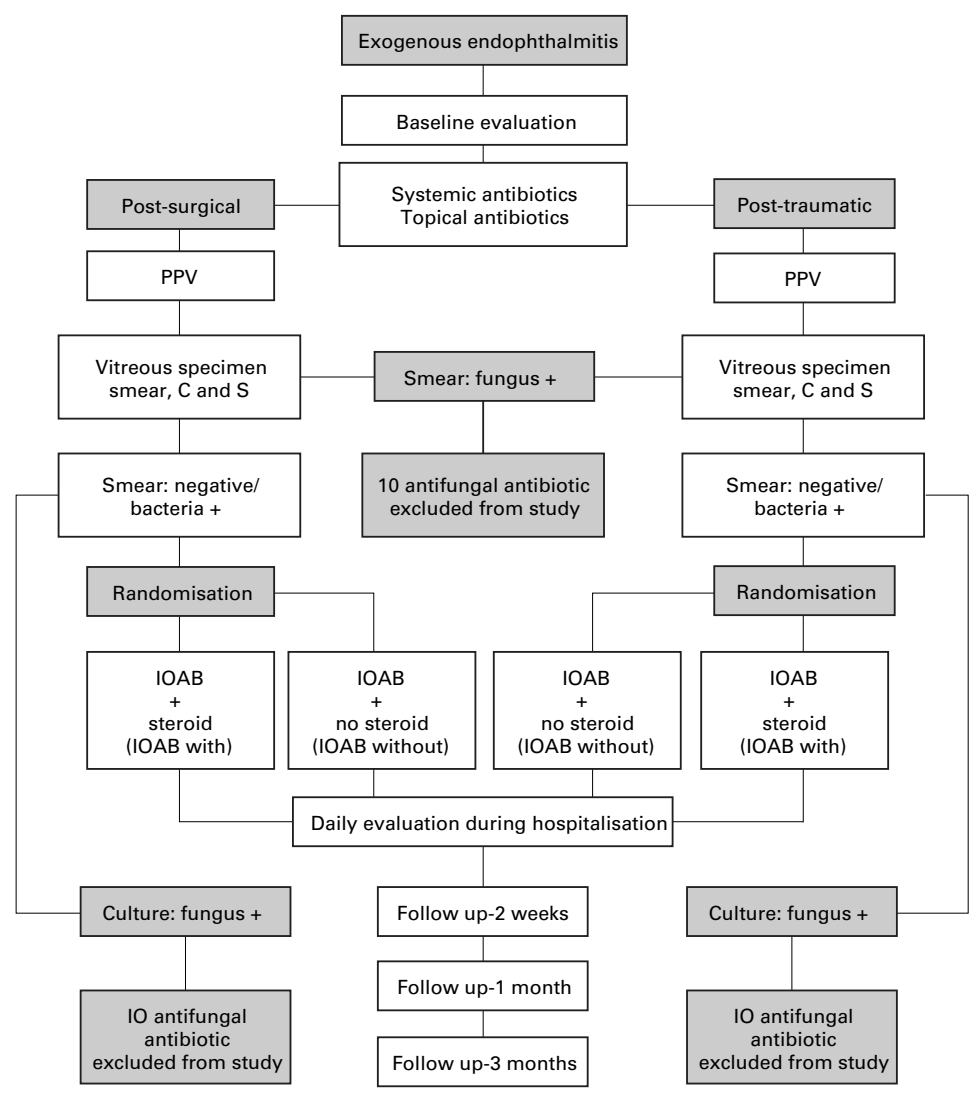

Figure 1 Study design.

ous intraocular surgery; visual acuity assessment, slit lamp biomicroscopy; fundus examination by indirect ophthalmoscope wherever the ocular media permitted, and B-scan ultrasonography otherwise. In most of the patients the vision was reduced to perceiving hand movements (HM), or light perception (LP) only. The LP was tested by shining the brightest light of the indirect ophthalmoscope directly into the eye; the HM was tested by shining the brightest light of the indirect ophthalmoscope from behind the patient's head while the hand with outstretched fingers was slowly moved from side to side. The projection of rays (PR) was tested by swinging the brightest light of the indirect ophthalmoscope from side to the centre. It was tested

Table 1 Quantitative endophthalmitis scoring scale

\begin{tabular}{|c|c|c|c|c|c|}
\hline \multirow[b]{2}{*}{ Tissue response } & \multicolumn{5}{|l|}{ Points } \\
\hline & 0 & 1 & 2 & 3 & 4 \\
\hline \multicolumn{6}{|l|}{ Cornea: } \\
\hline Clarity & Clear & Mild & $\begin{array}{l}\text { Moderate } \\
\text { (iris visible) }\end{array}$ & $\begin{array}{l}\text { Severe } \\
\text { (iris bare details) }\end{array}$ & $\begin{array}{l}\text { Opaque } \\
\text { (iris no view) }\end{array}$ \\
\hline Abscess & None & $<1 \mathrm{~mm}$ & $1-2 \mathrm{~mm}$ & $3-4 \mathrm{~mm}$ & $>5 \mathrm{~mm}$ \\
\hline \multicolumn{6}{|l|}{ Anterior chamber: } \\
\hline Flare/cells & None & Trace & Mild & Moderate & Severe \\
\hline Fibrin/hypopyon & None & $\begin{array}{l}\text { Mild } \\
<25 \%\end{array}$ & $\begin{array}{l}\text { Moderate } \\
>25 \%\end{array}$ & $\begin{array}{l}\text { Severe } \\
\text { details }\end{array}$ & No iris \\
\hline \multicolumn{6}{|l|}{ Iris: } \\
\hline Blood vessels & None & Mild & Moderate & Severe & NVI \\
\hline Exudates over & None & $\begin{array}{l}\text { Mild } \\
<25 \%\end{array}$ & $\begin{array}{l}\text { Moderate } \\
<50 \%\end{array}$ & $\begin{array}{l}\text { Severe } \\
<75 \%\end{array}$ & $\begin{array}{l}\text { Pupil } \\
\text { occluded }\end{array}$ \\
\hline \multicolumn{6}{|l|}{ Vitreous: } \\
\hline Flare & None & Trace & Mild & Moderate & Severe \\
\hline Opacities & None & cells & Clumps & Red reflex & Opaque \\
\hline
\end{tabular}

Cornea opaque, add 20; AC opaque, add 15; pupil fully covered with exudates, add 10; vitreous opaque, add 5. twice in each of the four principal cardinal directions. Slit lamp photodocumentation was done in all the patients preoperatively and at predetermined time frames postoperatively.

The study design consisted of treating all recruited patients with vitrectomy and IOAB. Then the eyes were randomised to "with" intravitreal dexamethasone (IOAB with) and to "without" intravitreal dexamethasone (IOAB without) treatment. The randomisation was done using the standard randomisation table; the sealed envelopes were opened by the circulating nurse just before the preparation of the IOABs. The initial IOAB combination used in this study was amikacin $(400 \mu \mathrm{g})$ and vancomycin (1 mg) in all eyes. The intravitreal dexamethasone, whenever injected, was $400 \mu \mathrm{g}$ in $0.1 \mathrm{ml}$. Both intravitreal antibiotics and dexamethasone were injected only after obtaining the vitreous biopsy microscopy report from the microbiology laboratory. The usual time taken for the microscopy report was 10-15 minutes. Whenever the microscopy report was positive for fungus, the eye was withdrawn from the study and treated using the institutional protocol of fungal endophthalmitis. Similarly, eyes with fungus positive culture were excluded from the study. All the eyes received subconjunctival antibiotics (same as the intravitreal antibiotics) at the end of the surgery; subconjunctival steroid was not given to any of the eyes in the study. All the patients also received intravenous antibiotics (gentamicin and cefazoline) for a period of 7 days.

\section{INFLAMMATION SCORING}

The inflammation scoring was based on the clinical picture of the cornea, anterior chamber, iris, and the vitreous. This quantitative inflammatory scoring used in the study was modified from the one used by Meredith et $a l^{4}$ (Table 1). The scoring was done from 0 to 4 in each category, with additional allowance given for poor clarity of ocular tissues. The inflammation score (IS) was done by two independent observers - one ophthalmologist and one senior optometrist, experienced with eye examination by slit lamp and indirect ophthalmoscope.

\section{SURGERY}

All the patients were operated within 8 hours of presentation. A standard three port, and occasionally two port, vitrectomy was done. A $6 \mathrm{~mm}$ infusion cannula in three port vitreous surgery, and angulated cannula (Wilson's cannula) in two port vitrectomies were used. Two port vitrectomy was performed in selected cases when a limited anterior vitrectomy only was considered necessary. Vitreous samples were collected in all cases before opening the infusion cannula so as to obtain an undiluted vitreous sample. Cataract extraction (lensectomy/lens fragmentation) or explantation of the intraocular lens (IOL) was done if considered an absolute necessity with prior informed consent. Lens extraction was considered necessary wherever the lens was injured in cases of traumatic endophthalmitis; the IOL was explanted in cases of acute endophthalmitis when the visibility of 
Table 2 Patient demography

\begin{tabular}{lll}
\hline \multirow{2}{*}{ Postoperative endophthalmitis } & \\
& IOAB without group $(n=16)$ & IOAB with group $(n=16)$ \\
Age & 64.5 years $(48-84)$ & 55.1 years $(40-74)$ \\
Sex & Males 11 & Males 11 \\
& Females 5 & Females 5 \\
Laterality & Right 8 & Right 8 \\
& Left 8 & Left 8 \\
Past surgery & ECCE, IOL 10 & ECCE, IOL 8 \\
& ECCE 3 & ECCE 5 \\
& ICCE 2 & ICCE 3 \\
Post-traumatic endophthalmitis & \\
& IOAB without group $(n=18)$ & \\
Age & 12.2 years $(1.5-33)$ & IOAB with group $(n=13)$ \\
Sex & Males 10 & 20.8 years $(08-45)$ \\
& Females 8 & Males 10 \\
Laterality & Right 9 & Females 3 \\
& Left 9 & Right 8 \\
IOFB & Nil & Left 5 \\
& & Nil
\end{tabular}

Table 3 Combined anatomical and functional success

\begin{tabular}{lllll}
\hline Event & $\begin{array}{l}\text { Culture } \\
\text { report }\end{array}$ & Group & $\begin{array}{l}\text { No of } \\
\text { subjects }\end{array}$ & $\begin{array}{l}\text { Rate success } \\
(\%)\end{array}$ \\
\hline Postop & Negative & IOAB with & 7 & $7(100)$ \\
& & IOAB without & 7 & $7(100)$ \\
& Positive & IOAB with & 9 & $8(88.88)$ \\
Post-trauma & Negative & IOAB without & 9 & $6(66.66)$ \\
& & IOAB with & 4 & $4(100)$ \\
& Positive & IOAB without & 5 & $2(40)$ \\
& & IOAB with & 9 & $6(66.66)$ \\
& & & 13 & $9(69.23)$ \\
& & &
\end{tabular}

the anterior chamber and the pupil was totally obscured by exudates and fibrin membranes. Following the vitrectomy, IOAB with or without intravitreal dexamethasone was injected.

Four patients in the postoperative group and six patients in the post-trauma group received a second injection of intravitreal antibiotics after the culture report was available. This was usually after 72 hours, and was culture adjusted. Intravitreal dexamethasone was not repeated.

\section{MICROBIOLOGICAL EVALUATION}

Undiluted vitreous specimen collected at the beginning of the vitreous surgery and the cassette fluid at conclusion of the surgery were the sources of samples of microbiological study. The undiluted $0.5 \mathrm{ml}$ vitreous was collected in a $2 \mathrm{ml}$ disposable syringe attached to vacuum line of the vitreous cutter and vacuum was created by mechanical suction exerted by the surgical assistant. The syringe with the vitreous sample was then capped with a hypodermic needle; the needle was either bent or plunged into a sterile rubber cork before being transported in a sterile plastic container to the microbiology laboratory of the institute. The cassette used in the surgery was transported to the microbiology laboratory soon after the conclusion of surgery.

The microbiological investigations were done using standard protocol of the institute. ${ }^{9}$

Table 4 Average inflammation score

\begin{tabular}{lllll}
\hline Group & Preop & 1 Week & 4 Weeks & 12 Weeks \\
\hline IOAB with & $19.3(5.9)$ & $\begin{array}{l}16.4(8) \\
(\mathrm{p}<0.0001)^{\star}\end{array}$ & $\begin{array}{l}10.9(8.7) \\
(\mathrm{p}<0.0001)^{\star}\end{array}$ & $\begin{array}{l}2.6(2.2) \\
(\mathrm{p}<0.0001)^{\star}\end{array}$ \\
IOAB without & $18.6(5.3)$ & $\begin{array}{l}21.6(8.1) \\
(\mathrm{p}=0.009)^{\star \star}\end{array}$ & $\begin{array}{l}14.0(8.4) \\
(\mathrm{p}=0.001)^{\star}\end{array}$ & $\begin{array}{l}3.2(2.8) \\
(\mathrm{p}<0.0001)^{\star}\end{array}$ \\
\hline
\end{tabular}

* Significant decrease in inflammation score.

${ }^{\star}$ Significant increase in inflammation score.

(Paired $t$ test.)
Briefly it consisted of examination of the vitreous biopsy smear (Gram, Giemsa, and Calcofluor white stains) and culture. The cassette fluid was passed through a Millipore filter $(0.22 \mu \mathrm{m})$, aseptically cut into six pieces, and cultured. The culture was done for both aerobic and anaerobic organisms using both solid and liquid media. The media used for culture included sheep blood agar, chocolate agar, brain-heart infusion, thioglycolate broth, Sabouraud's dextrose agar, and potato dextrose agar. The sensitivity to antibacterial antibiotics were done by standard disc diffusion technique. ${ }^{10}$ The culture was interpreted using the guidelines of the EVS study. ${ }^{11}$

\section{POSTOPERATIVE CARE}

Following the vitrectomy and intravitreal drug therapy, intravenous and topical antibiotics, topical steroids, and cycloplegics were continued. The topical antibiotics and steroids were instilled hourly during waking hours (usually from 6 am to $10 \mathrm{pm}$ ), and atropine three times a day. The topical antibiotics and steroids were reduced to 2 hourly after 2 weeks and further reduced progressively after 4 weeks and discontinued after 8 weeks.

The clinical evaluation in the first postoperative week consisted of estimation of visual acuity, slit lamp biomicroscopy and indirect ophthalmoscopy twice daily, and slit lamp photography on the first and seventh postoperative day. The follow up visits were scheduled at second week, fourth week, third month, and sixth month. At each evaluation the visual acuity, cornea (clarity), the anterior chamber (flare, cells, hypopyon, fibrin), the iris (blood vessels, exudates), and vitreous (cells, opacities) were evaluated and documented. The IS was done by same two independent observers to quantitate the degree of inflammation at each evaluation.

Because of known renal toxicity of aminoglycosides, baseline blood urea, and serum creatinine was evaluated, and repeated 5 days later. Statistical analysis for paired comparison was done using paired $t$ test, and for group comparison of normal data using Student's $t$ test and Mann-Whitney U for grouped comparison of non-normal data.

\section{Results}

DEMOGRAPHY

Sixty eight consecutive patients were recruited into the study. Five patients (two postoperative and three post-traumatic) were excluded as the initial vitreous smear or the final vitreous culture was positive for fungus. In four of these eyes, the vitreous biopsy microscopy had demonstrated filamentous fungus filaments; these patients were excluded at the beginning of the study. In one patient with traumatic endophthalmitis intravitreal dexamethasone was injected, but the final culture was positive for fungus; this patient, however, did not show any adverse reaction.

Out of 63 patients, 32 patients had endophthalmitis following intraocular surgery, and 31 patients had endophthalmitis after trauma. After randomisation 16 patients in the postop- 

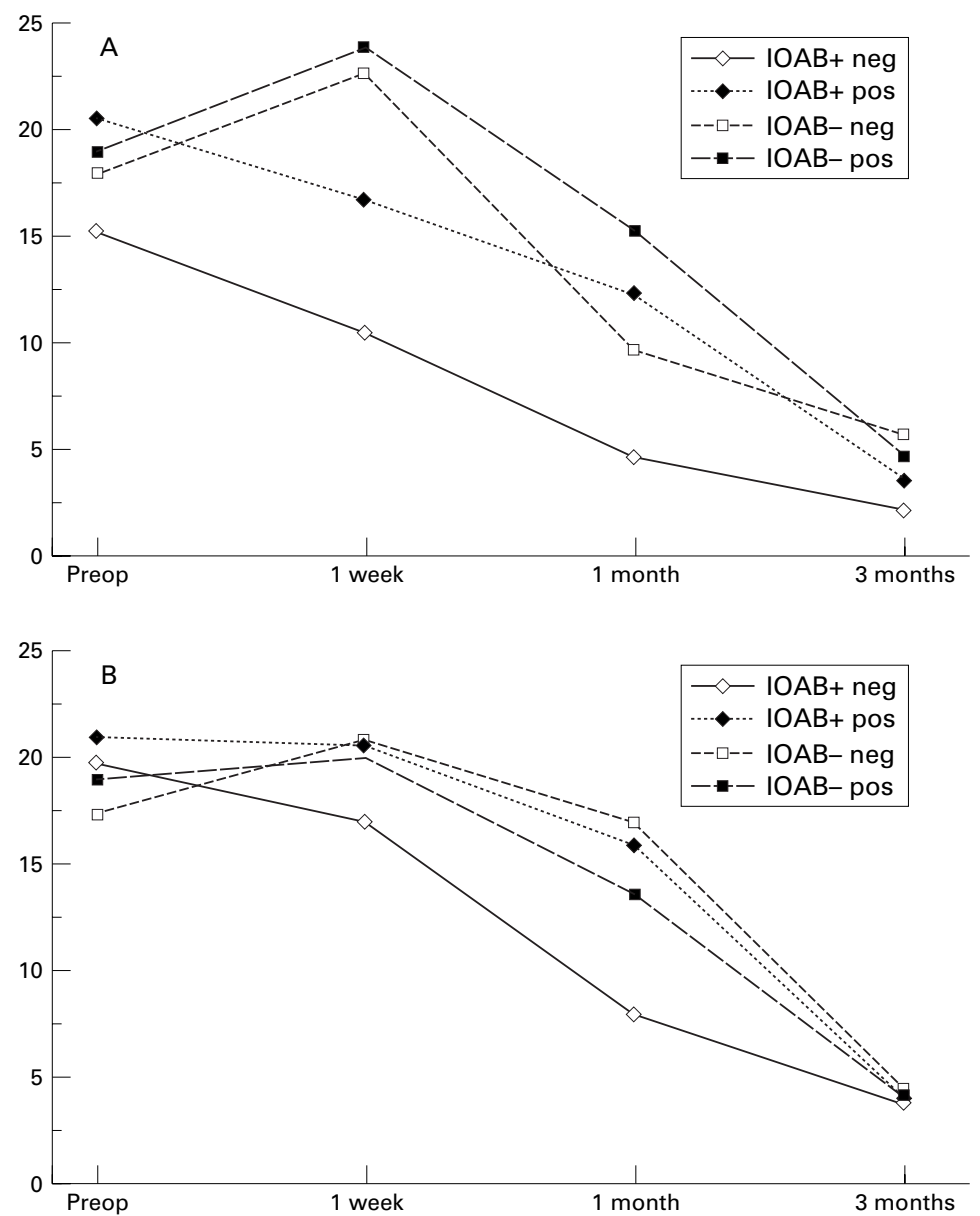

Figure 2 Average inflammation score. (A) Postoperative endophthalmitis. (B) Post-traumatic endophthalmitis.

Table 5 Magnitude of change of inflammation score

\begin{tabular}{llll}
\hline Time point & $\begin{array}{l}\text { IOAB with IS } \\
\text { (SD) (median) }\end{array}$ & $\begin{array}{l}\text { IOAB without IS } \\
\text { (SD) (median) }\end{array}$ & p Value \\
\hline 1 Week & $2.9(3.6)(3)$ & $-3.0(6.4)(-4)$ & $<0.0001^{\star}$ \\
4 Weeks & $8.3(6.2)(9)$ & $4.6(6.9)(6)$ & $<0.0110^{\star}$ \\
12 Weeks & $16.7(6.1(16)$ & $15.4(6.5)(14)$ & 0.3831 \\
\hline
\end{tabular}

*Statistically significant difference (Mann-Whitney U test).

erative group and 13 patients in the posttraumatic group received intraocular antibiotics with dexamethasone (IOAB with). The remaining 16 and 18 patients in the postoperative and post-traumatic group respectively received only intraocular antibiotics without dexamethasone (IOAB without). The demographic details of the patients are shown in Table 2.

MICROBIOLOGY

Culture was positive in 39 out of 63 patients $(61.90 \%)$ in this study. This included 18 patients in the postoperative group $(56.25 \%)$ and 21 in the post-traumatic group $(67.74 \%)$.

Table 6 Relative percentage of change in inflammation

\begin{tabular}{llll}
\hline Time period & $\begin{array}{l}\text { IOAB with IS \% } \\
\text { (SD) (median) }\end{array}$ & $\begin{array}{l}\text { IOAB without IS } \% \\
\text { (SD) (median) }\end{array}$ & p Value \\
\hline 1 Week & $19.2(26.0)(20)$ & $-18.3(35.7)(-23.9)$ & $0.0001^{\star}$ \\
4 Week & $47.5(35.8)(56.5)$ & $26.0(37.7)(33.8)$ & $0.0037^{\star}$ \\
12 Weeks & $85.9(11.3)(84.2)$ & $81.0(16.2)(78.0)$ & 0.1863 \\
\hline
\end{tabular}

^Statistically significant (Mann-Whitney U test).
POSTOPERATIVE STATUS

The anatomical success was defined as maintenance of the shape of the eye ball with intraocular pressure at least $5 \mathrm{~mm} \mathrm{Hg}$, and functional success as visual acuity of at least $6 / 120$. In the postoperative endophthalmitis "IOAB without" group $(n=16)$ both anatomical and functional success could be achieved in 13 out of 16 patients $(81.5 \%)$. Three eyes were phthisicaltwo eyes were infected with $S$ pneumoniae, and one eye with $P$ aeruginosa. In the postoperative "IOAB with" group $(\mathrm{n}=16)$ favourable outcome was seen in 14 eyes $(87.5 \%)$. Two eyes were phthisical and in both the events it was $S$ pneumoniae endophthalmitis.

In the post-traumatic endophthalmitis IOAB without group $(n=18) \quad 11$ eyes $(61.11 \%)$ benefited. The visual acuity could not be assessed in one patient because of very young age ( 1.5 years). Seven eyes were phthisical and in three of them the vitreous was culture negative. In the post-traumatic endophthalmitis IOAB with group $(n=13) 10$ patients benefited $(76.92 \%)$. Three eyes went into phthisis and all of them had Bacillus endophthalmitis. The details of anatomical and functional success in various groups is shown in Table 3.

\section{INFLAMMATION SCORING}

The mean preoperative inflammation score (IS) was 19.3 (SD 5.9) in the IOAB with group, and 18.6 (5.3) in the IOAB without group. These values were not significantly different $(p=0.611)$. Statistically significant reduction $(\mathrm{p}<0.0001)$ in IS at 1,4 , and 12 weeks following surgery was seen in the IOAB with group. Significant increase $(p<0.01)$ in IS at 1 week following surgery was noted in the IOAB without group; however, there was significant reduction $(\mathrm{p}<0.001)$ in postoperative IS at 4 and 12 weeks (Table 4; Fig 2). The magnitude and relative percentage of change in IS between the IOAB with and without groups showed significant reduction at 1 and 4 weeks only, and not at 12 weeks (Tables 5 and 6 ).

\section{Discussion}

Corticosteroids are widely used in the treatment of inflammatory diseases. The primary value of corticosteroids in ocular conditions has been to minimise the inflammatory response concurrently preserving the anatomical structure of the ocular tissue. It is believed that concurrent administration of corticosteroids and antibiotics is necessary in bacterial endophthalmitis to control both infection and inflammation. ${ }^{12}{ }^{13}$ In the $\mathrm{EVS}^{8}$ oral predinisolone was administered for 5-10 days. Considering that on occasions oral administration of steroid could be relatively or absolutely contraindicated, this study was designed to address whether intravitreal steroid could replace oral administration, and be complementary to oral administration. The secondary aim was to study concurrently if it has any measurable effect on long term visual benefit.

Generally, factors that contribute to severity of bacterial infections include secreted toxins and cell surface components that result in direct tissue damage, induction of host inflam- 
matory response, or both. Intraocular infections are frequently rapid and severe because of the avascular nature of the intraocular space that allows early stages of bacterial infection to proceed without significant host response. Severe inflammation associated with endophthalmitis is known to cause breakdown of the blood-retinal barrier ${ }^{14}$ which presumably plays an important part in the final outcome.

The inflammation is modulated by toxins liberated by invading organisms and injury to the ocular tissue caused in the process. The effect of toxins in determining the course and severity of some of the micro-organisms such as Enterococcus faecalis, Staphylococcus aureus, and Escherichia coli induced endophthalmitis are already documented. ${ }^{15-17}$ While similar studies for the toxins of other bacteria associated with fulminant and destructive endophthalmitis are not yet reported, it is speculated that toxin production by the bacteria contributes to the poor prognosis in endophthalmitis. ${ }^{18}$ It is also being speculated that treatment of Gram negative endophthalmitis with antibiotics alone can result in release of inflammatory endotoxin, thereby exacerbating inflammation mediated ocular damage. ${ }^{5}$ In addition, the host inflammatory response makes a measurable contribution such as intraocular fibrocellular proliferation, membrane formation, and traction retinal detachment. This calls for aggressive management to limit both visual loss and severe ocular damage. ${ }^{19}$

In several earlier reports intravitreal dexamethasone has been found to be a safe and effective adjunct to a combination of broad spectrum antibiotics for suppressing the inflammatory response during treatment of bacterial endophthalmitis. ${ }^{1-6}$ Graham and Peyman ${ }^{1}$ observed a significant reduction in inflammatory response in experimental Pseudomonas infection when the eyes were treated with intravitreal dexamethasone and gentamicin within 5 hours of infection. Irvine et $a l,{ }^{6}$ on retrospective evaluation of Gram negative endophthalmitis, observed that the eyes treated with both intravitreal antibiotic and dexamethasone had ultimately better visual acuity compared with the eyes treated with intravitreal antibiotic alone.

Intravitreal dexamethasone does not seem to have any detrimental effect on the efficacy and potency of the intravitreal antibiotics and a dose of $400 \mu \mathrm{g}$ is non-toxic to the retina. ${ }^{20}$ Doses higher than $800 \mu \mathrm{g}$ are known to cause vacuolation between the outer plexiform and outer nuclear layers and the primary site of toxic reaction is the Müller cells. The potential advantages of co-administration of antibiotics and dexamethasone include reduction of the harmful effects of inflammation within the eye to preserve the integrity of retina and other ocular tissues. The potential disadvantages include the possibility of suppressing mechanisms for infection control and the risk of drug toxic reaction from steroid, either alone or in combination with antibiotics.

A recommendation has been made previously that anti-inflammatory therapy for infectious endophthalmitis be postponed for 12 hours after antibiotic administration to allow for efficient killing of the offending organism. ${ }^{22}$ However, experimental evidence does not suggest any additional beneficial effect of prior or delayed administration of intravitreal dexamethasone. ${ }^{15}$ In experimental models of Staphylococcus epidermidis endophthalmitis increasing signs of inflammation have been observed for first 5-7 days. ${ }^{4}$ This suggests that dying bacteria and products of infection produce continuing inflammation even in the absence of active bacterial replication. Hence, it is expected that control of the inflammatory component in the infectious process may contribute to a favourable outcome.

Many organisms (such as Bacillus cereus, Staphylococcus aureus, Streptococcus species, Clostrodium species, and Pseudomonas species) liberate potent toxins and tissue damaging enzymes that may contribute to virulence of intraocular infection. ${ }^{23}{ }^{24}$ The treatment of infections with such organisms poses serious problems, because even if the intraocular spaces are sterilised with appropriate antibiotic(s), a significant amount of bacterial debris and potentially toxic products remain to account for treatment failure. Hence the treatment of bacterial endophthalmitis should be simultaneously directed to control of both infection and inflammation.

The results of this study show that there is an early resolution (first week) of inflammation in both culture positive and negative endophthalmitis with intravitreal steroid administration; however, the final visual acuity at the end of the third month is independent of intravitreal steroid therapy. Since maximum and increasing signs of inflammation are reportedly apparent in the first 5-7 days following infection, ${ }^{4}$ co-administration of antibiotics and dexamethasone into the primary site of infection (vitreous) appears logical. Probably, containment of inflammation helps the eye to recover faster and maintain the structural integrity of the globe. Additionally, the antiinflammatory drugs reduce the fibrinous reactions in the eye. Intravitreal injection of dexamethasone is a safe and effective route of drug delivery without the complications of systemic corticosteroid therapy. A serious limitation of intravitreal dexamethasone therapy is that it is cleared from the vitreous cavity in 3 days $^{21}$ thus denying a prolonged beneficial effect of such therapy. Since the maximum inflammatory damage to the eye in bacterial endophthalmitis occurs in the first few days, intravitreal dexamethasone is useful and could be the sole route of administration in patients when oral corticosteroids are contraindicated for medical reasons. In other situations both intravitreal and oral corticosteroids could be complementary.

In this study both postoperative and posttraumatic endophthalmitis patients are pooled together though they are analysed separately. Conceding that more virulent organisms are associated with traumatic endophthalmitis, and often with more severe associated ocular damage, the study none the less demonstrates the beneficial effects of intravitreal dexamethasone. Small sample size is also an inherent weakness of this study, but even so the primary questions of the study are adequately an- 
swered. Based on these study results our suggestion has been to use both oral and intravitreal steroid in bacterial endophthalmitis, and intravitreal steroids only when oral corticosteroids are absolutely contraindicated.

Presented in part at the American Academy of Ophthalmology Annual Meeting, Chicago October,1996 and Association for Research in Vision and Ophthalmology Annual Meeting, Ft Lauderdale, May 1997.

Supported by grants from the Hyderabad Eye Research Foundation, Hyderabad, India.

The authors have no proprietary interest in any of the materials used in this study.

1 Graham RO, Peyman GA. Intravitreal injection of dexamethasone: treatment of experimentally induced endophmethasone: treatment of experimentally in

2 Peyman GA, Herbst R. Bacterial endophthalmitis: treatment with intraocular injection of gentamicin and dexament with intraocular injection of gentamicin

3 Diamond JG. Intraocular management of endophthalmitis: a systemic approach. Arch Ophthalmol 1981;99:96-9.

4 Meredith TA, Aguilar E, Miller MJ, et al. Comparative treatment of experimental Staphylococcus epidermidis endophthalmitis. Arch Ophthalmol 1990;108:857-60. 5 Schulman JA, Peyman GA. Intravitreal corticosteroids as an
adjunct in the treatment of bacterial and fungal endophthalmitis: a review. Retina 1992;12:336-40.

6 Irvine WD, Flynn HW, Miller D, et al. Endophthalmitis caused by gram negative organism. Arch Ophthalmol 1992; 110:1450-4.

7 Doft BH. The endophthalmitis vitrectomy study. Arch Ophthalmol 1991;109:487-9.

8 Endophthalmitis Vitrectomy Study Group. Results of the Endophthalmitis Vitrectomy Study. Arch Ophthalmol 1995; 113:1479-96.

9 Sharma S, Jalali S, Adiraju MV, et al. Sensitivity and predictability of vitreous cytology, biopsy, and membrane filter ability of vitreous cytology, biopsy, and membran

10 Bauer AW, Kirby WMM, Sherris JC, et al. Antibiotic susceptibility testing by a single disc method. Am $\mathcal{F}$ Clin Pathol 1966;45:493.
11 Han DP, Wisniewski SR, Wilson LA, et al and Endophthalmitis Study Group. Spectrum and susceptibility of microbiologic isolates in the Endophthalmitis Study Group. Am f Ophthalmol 1996;122:1-17.

12 Das T, Dogra MR, Gopal L, J et al. Postsurgical endophthalmitis: diagnosis and management. Indian $\mathcal{f}$ Ophthalmol 1995;43:103-16.

13 Gardner S.Treatment of bacterial endophthalmitis. Part I. Ocular therapeutics and Management 1992;2:1.

14 Metrikin DC, Wilson CA, Berkowitz BA, et al. Measurement of blood-retinal barrier breakdown in endotoxininduced endophthalmitis. Invest Ophthalmol Vis Sci 1995; 36:1361-70.

15 Jet BD, Jennies HG, Atkuri RV, et al. Evaluation of therapeutic measures for treating endophthalmitis caused by isogenic toxin producing and toxin-nonproducing Enterococcus faecalis strains. Invest Ophthalmol Vis Sci 1995;36: rococcus

16 Booth MC, Atkuri RV, Nanda SK, et al. Accessory gene regulator controls Staphylococcus aureus virulence in endophthalmitis. Invest Ophthalmol Vis Sci 1995;36:182836.

17 Jacobs DR, Cohen HB. The inflammatory role of endotoxin in rabbit Gram-negative bacterial endophthalmitis. Invest Ophthalmol Vis Sci 1984;25:1074-9.

18 Baum J. Therapy for ocular bacterial infection. Trans Ophthalmol Soc UK 1986;105:69-77.

9 Bustros SD, Michels RG, Glaser BM. Evolving concepts in the management of posterior segment penetrating ocular injuries. Retina 1990;10(suppl):s72-5.

20 Charp AD. In: Tasman W, Jaeger EA, eds. Duane's foundations of clinical ophthalmology. Philadelphia: JB Lippincott, chapter 31:1:35.

21 Kwak HW, D'Amico DJ. Evaluation of the retinal toxicity and pharmacokinetics of dexamethasone after intravitreal injection. Arch Ophthalmol 1992;110:259-66.

22 Smolin G, Tabbara K, Whitcher J. Infectious diseases of the eye. Baltimore: Williams and Wilkins, 1984:148-61.

23 Davey RJ, Tauber WB. Posttraumatic endophthalmitis: the emerging role of Bacillus cereus infection. Rev Infect Dis 1987;9:110-23.

24 Engstrom RE Jr, Mondino BJ, Glasgow BJ, et al. Immune response to Staphylococcus aureus endophthalmitis in rabbit model. Invest Ophthalmol Vis Sci 1991;32:181-6. 